\title{
C-kit proto-oncogene expression in uterine leiomyosarcomas: case series
}

Naik VR and Hasnan J

Department of Pathology, Universiti Sains Malaysia, Kubang Kerian, Kelantan, Malaysia.

\begin{abstract}
Introduction: The proto-oncogene c-kit is the cellular homologue of the oncogene v-kit of HZ4 feline sarcoma virus. It is located on chromosome 4 (4q11-12) in the human genome. Interaction between the c-kit receptor and its ligand, stem cell factor, is essential in the development of tissues. C-kit expression has been identified in a number of different neoplasms like seminoma/dysgerminoma, and gastrointestinal stromal tumors (GIST). Recently it has been reported that c-kit is also present in leiomyosarcomas. Tyrosine kinase inhibitors (TKIs) are a promising new therapy in the treatment of cancer. These agents target cellular proteins like kit and its related homologues decreasing cellular proliferation and survival. TKIs may be helpful in treating leiomyosarcomas expressing c-kit. Materials and Methods: In this study a total of 6 cases diagnosed as leiomyosarcomas at Department of Pathology, Universiti Sains Malaysia, Kubang Kerian, Malaysia, were investigated for reactivity for c-kit using immunohistochemical stain. Stain was considered positive if more than 10 percent of the cells showed membrane or cytoplasmic positivity. Results: Two leiomyosarcomas stained faintly with c-kit and in less than 10 percent of the cells. The other 4 cases showed no staining. The control showed good membrane and cytoplasmic positivity. Conclusion: Uterine leiomyosarcomas did not express c-kit. The reason for this could be that the tumors are inherently c-kit negative. More study using larger number of cases is required to validate these findings and further molecular characterization of these mesenchymal tumors is needed to identify the true nature of these sarcomas.
\end{abstract}

KEYWORDS: Leiomyosarcoma, Uterus, C-kit protein, Immunohistochemistry.

\section{INTRODUCTION}

The proto-oncogene c-kit is the cellular homologue of the oncogene v-kit of HZ4 feline sarcoma virus. It is located on chromosome $4(4 q 11-12)$ in the human genome. The gene encodes for a $145-\mathrm{kDa}$ transmembrane tyrosine kinase receptor (KIT), which is immunologically identified by the CD117 antigenic epitope. Binding of Stem cell factor induces KIT dimerisation and autophosphorylation of specific tyrosine residues. This leads to activation of several signal transduction pathways important for cell growth and survival.

Expression of c-kit is present in haematopoietic stem cells, mast cells, gametocytes, melanocytes, and interstitial cells of Cajal located in the intestinal tract. Interaction between the c-kit receptor and its ligand, stem cell factor, is essential in the development of these tissues. KIT is not expressed in normal squamous epithelium, glandular epithelium of endocervix, pancreas, prostate and intestines.

Corresponding address

Venkatesh R.Naik

Department of Pathology

School of Medical Sciences

Universiti Sains Malaysia

Kubang Kerian

Malaysia.
Somatic mutations of c-kit causing constitutive activation of KIT have been found in a number of different neoplasms. The list includes mastocytosis/mast cell leukaemia, acute myeloblastic leukaemia, seminoma/dysgerminoma, and gastrointestinal stromal tumors (GIST).

Tyrosine kinase inhibitors (TKIs) are a promising new therapy in the treatment of cancer. These agents target cellular proteins like kit and its related homologues decreasing cellular proliferation and survival. They have been used successfully in the treatment of bcr-abl-positive leukaemia's and GIST.

The knowledge on c-kit expression in uterine mesenchymal tumors is presently unclear. There have been conflicting reports by various studies. Identification of c-kit expression in these tumors could lead to therapeutic options that employ TKI's and the detection of specific mutations that could better refine this potential treatment.

The uterine leiomyosarcomas are rare sarcomas arising from the smooth muscle cells found within the myometrium. They constitute $25 \%$ of all uterine sarcomas and $1 \%$ of all uterine corpus malignant tumours. The median age of presentation is 54 years and they usually present typically with vaginal bleeding, pain, and a pelvic mass. Atypical presentations with hypercalcemia or eosinophilia have been reported. They exhibit histologic features similar to that observed in 
soft tissue leiomyosarcomas. The behaviour and treatment, however, markedly differs. Most cases arise de novo and not from pre-existing leiomyomas, which are benign smooth muscle tumors and are very common. Their resistance to chemotherapy and radiation treatment characterizes these tumours. Surgery is the primary method of treatment, but for patients with unresectable disease, alternate therapeutic options are clearly warranted.

In view of this, active research has been undertaken to find better ways of managing this malignancy.

\section{MATERIALS AND METHODS}

Six cases of uterine leiomyosarcomas were retrieved from the archives of the Department of Pathology at Universiti Sains Malaysia from 1996 to 2004. These cases did not include the cases referred to the pathology department from other hospitals.

Haematoxylin and Eosin stained sections of the formalin fixed paraffin embedded tissue sections of these cases were examined to reconfirm the diagnosis and to select the most appropriate for performing the immunohistochemical stain based on the amount of viable well preserved tumour tissue seen in the sections.

Four-micron thick sections were again taken, deparaffinised and incubated in 3\% hydrogen peroxide for 10 minutes to remove endogenous tissue peroxidase. They were subjected to epitope antigen retrieval by putting the slides in Tris- EDTA buffer having a pH of 9.00 and placing them for a total period of 14 minutes in a microwave oven. Then the slides were rinsed in TBS buffer at a pH of 7.4 for 2 minutes. The slides were then treated with an anti-c-kit9 [CD117] polyclonal antibody [DAKO, 1:400 dilutions]. Avidin-bioton-peroxidase detection kit was used and 3'3'-diaminobenzidene $[\mathrm{DAB}]$ was used to develop the immunohistochemical stain. All the sections were counterstained with haematoxylin and mounted. GIST tumour was used as positive control and stratified squamous epithelium was used as negative control.

The stain was considered positive if there was a cytoplasmic and / or membrane staining present. The slide was considered to be positive for c-kit if more than $10 \%$ of the cells showed this type of staining.

\section{RESULTS}

The six cases diagnosed as leiomyosarcoma belonged to the age group of 47 to 67 year old patients and all of them were Malays.

Of the six cases stained 2 cases stained faintly for c-kit in less than 10 percent of the cells and the rest were negative without even 10 percent of the cells staining for c-kit.

\section{DISCUSSION}

There has been only a hand full studies documenting the immunohistological expression of CD117 in the female genital tract. Majority of these publications have focussed on epithelial lesions including endometrial hyperplasia, endometrial carcinoma and ovarian carcinoma. The expression of CD117 has been shown in normal proliferative and secretory endometrium as well as in endometrial hyperplasia. ${ }^{1}$ However there are a few studies that have reported negative immunostaining for CD117 in endometrium. ${ }^{2,3,4}$

There are several publications demonstrating the utility of c-kit in identification and diagnosis of GISTs. Moreover the study of these properties has led to the development of new treatment modalities targeting the KIT protein as a tyrosine kinase receptor, which is active in majority of GISTs. The kit protein is selectively and competitively bound by a newly developed small molecule, STI-571, resulting in inhibition of tyrosine phosphorylation. This shuts down the transcriptional activity of the cells resulting in growth arrest. $^{5}$

Several gains of function mutations in c-kit gene have been uncovered. Mutations in the juxtamembrane domain [exon11] have been reported to be the most frequent mutations in solitary GISTs and these c-kit mutations have been associated with malignant behaviour more frequently than those in which mutations have not been identified. ${ }^{5}$ However, the clinical role of other c-kit mutations in predicting the behaviour of GISTs is not known.

In a multicenter clinicopathologic study conducted by Mayerhofer et al, they concluded that leiomyosarcomas having vascular space involvement and having high mitotic count had a bad prognosis and presence of tumour necrosis and advanced stage of the disease has poorer prognosis. ${ }^{1,6}$ No clinical factor had a statistically significant association with c-kit expression. ${ }^{7}$ Patients expressing c-kit have an aggressive tumour and belong to the older age group. ${ }^{7}$

Given the unprecedented success of STI 571, investigators are screening a variety of neoplasms using CD117 immunostaining. However the expression of CD1117 immuostaining in the mesenchymal lesions of the female genital tract is less studied. There are various studies, which have revealed both positive and negative staining of CD117 in leiomyosarcomas. ${ }^{1,8-10}$ Leath $\mathrm{CA}$ et al have reported that the intensity of staining for c-kit in some of the sarcomas of the uterus was stronger than in GISTs. ${ }^{12}$ In the study by Wang et al they reported that c-kit was positive in 75 percent of the leiomyosarcomas and the staining was of high intensity and diffuse. ${ }^{9}$ They also reported that highgrade endometrial stromal sarcomas displayed c-kit positivity. Klein WM and Kurman RJ have reported that none of the four leiomyosarcomas stained positive for c-kit and that mesenchymal tumours of the uterus and ovary rarely express c-kit. ${ }^{1}$ 
One of the study reported that c-kit is expressed in uterine leiomyosarcomas but there was no mutations in exon 11 or 17 as is seen in GIST. ${ }^{11}$ They said that some tumours, which express c-kit, might have mutations in exon 17 and theses tumors do not respond to imatinib mesylate. ${ }^{11}$ They concluded that though leiomyosarcomas were positive for c-kit they would not respond to treatment with tyrosine kinase receptors inhibitors. These findings have been further substantiated in a study conducted by Raspollini et al, who also found no mutations on exon 11 on genetic assessment. ${ }^{13}$ They reported 53.1 percent of leiomyosarcomas being c-kit positive and majority of these cases had advanced stage III or IV disease.

There is also no reported high-frequency microsatellite instability in leiomyosarcomas but there is loss of heterozygosity for chromosome 11 seen in some of the cases..$^{13}$ There have been indications that c-kit staining depended on the antibody used and also its dilutions but in our study antibodies from DAKO were used, which have been used by many other authors and the manufacturer has recommended the dilution used. ${ }^{14}$

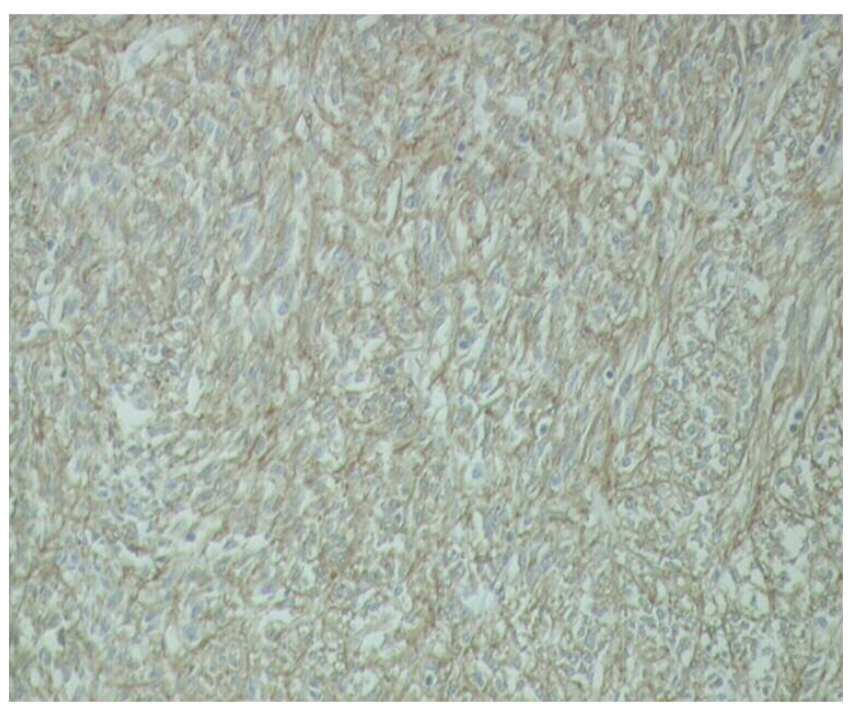

Fig 1: GIST used as control, CD117 immunostain, X40,
Fig 2: Focal positivity for CD117 immunostain seen in H\&E stain.

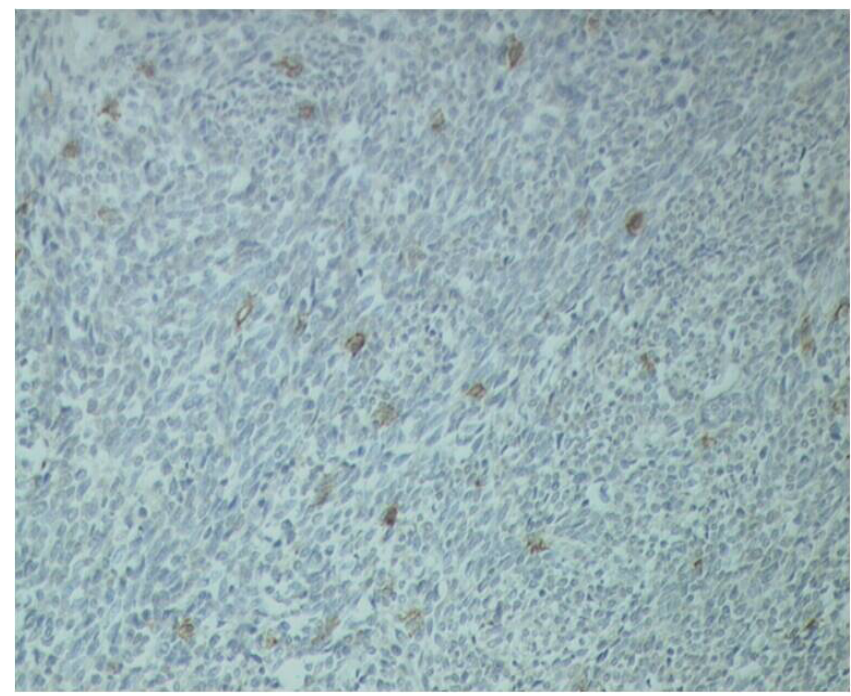

Leiomyosarcoma ,X10, H\&E stain.

\section{REFERENCES}

1. Klein WM, Kurman RJ. Lack of expression of c-kit protein (CD117) in mesenchymal tumors of the uterus and ovary. Int J Gynecol Pathol 2003; 22:181-4

2. Inoue M, Kyo S, Fujita M, Enomoto T, Kondoh $\mathrm{G}$. Coexpression of the c-kit receptor and the stem cell factor in gynecological tumors. Cancer Res 1994; 54:3049-53

3. Matsuda R, Takahashi T, Nakamura S, Sekido $Y$, Nishida K, Seto M, Seito T, Sugiura T, Ariyoshi $\mathrm{Y}$, Takahashi T, et al. Expression of the c-kit protein in human solid tumors and in corresponding fetal and adult normal tissues. Am J Pathol 1993;142:339-46

4. Natali PG, Nicotra MR, Sures I, Santoro E, Bigotti A, Ullrich A. Expression of c-kit receptor in normal and transformed human nonlymphoid tissues. Cancer Res 1992; 52:6139-43

5. Gibson PC, Cooper K. CD117 (KIT): a diverse protein with selective applications in surgical pathology. Adv Anat Pathol 2002; 9:65-9

6. Mayerhofer K, Obermair A, Windbichler G, Petru E, Kaider A, Hefler L, Czerwenka K, Leodolter S, Kainz C. Leiomyosarcoma of the uterus: a clinicopathologic multicenter study of 71 cases. Gynecol Oncol 1999; 74:196-201

7. Winter WE 3rd, Seidman JD, Krivak TC, Chauhan S, Carlson JW, Rose GS, Birrer MJ. Clinicopathological analysis of c-kit expression in carcinosarcomas and leiomyosarcomas of the uterine corpus. Gynecol Oncol 2003; 91:3-8

8. Chin-Hsiung Hsieh, Hao Lin, Chao-Cheng Huang, Eng-Yen Huang, Shiuh-Young Chang and Chan-Chao ChangChien. Leiomyosarcoma of the uterus: a clinicopathologic study of 21 cases. Acta Obstet Gynecol Scand 2003; 82: 74-81 
9. Wang L, Felix JC, Lee JL, Tan PY, Tourgeman DE, O'Meara AT, Amezcua CA. The proto-on cogene c-kit is expressed in leiomyosarcomas of the uterus. Gynecol Oncol 2004; 93:718

10. Rushing RS, Shajahan S, Chendil D, Wilder $\mathrm{JL}$, Pulliam J, Lee EY, Ueland FR, van Nagell $J R$, Ahmed MM, Lele SM. Uterine sarcomas express KIT protein but lack mutation(s) in exon 11 or 17 of c-KIT. Gynecol Oncol 2003; 91:9-14

11. Leath CA 3rd, Straughn JM Jr, Conner MG, Barnes MN 3rd, Alvarez RD, Partridge EE, Huh WK. Immunohistochemical evaluation of the c-kit proto-oncogene in sarcomas of the uterus: a case series. J Reprod Med 2004; 49:71-5

12. Raspollini MR, Amunni G, Villanucci A, Pinzani P, Simi L, Paglierani M, Taddei GL. C-Kit expression in patients with uterine leiomyosarcomas: a potential alternative therapeutic treatment. Clin Cancer Res 2004; 10:3500-3

13. Raspollini MR, Paglierani M, Taddei GL, Villanucci A, Amunni G, Taddei A. The protooncogene C-KIT is expressed in leiomyosarcomas of the uterus. Gynecol Oncol 2003; 90:402-6 